\section{L'Actualité économique}

L'ACTUALITÉ ÉCONOMIQUE

Essays on Economic Decisions Under Uncertainty, par JACQUES H. DRËZE. - Cambridge University Press, 1987, 424 p.

\section{Georges Dionne}

Volume 63, numéro 2-3, juin-septembre 1987

Incertain et information

URI : https://id.erudit.org/iderudit/601423ar

DOI : https://doi.org/10.7202/601423ar

Aller au sommaire du numéro

Éditeur(s)

HEC Montréal

ISSN

0001-771X (imprimé)

1710-3991 (numérique)

Découvrir la revue

Citer ce compte rendu

Dionne, G. (1987). Compte rendu de [Essays on Economic Decisions Under Uncertainty, par JACQUES H. DRÈZE. — Cambridge University Press, 1987, 424 p.] L'Actualité économique, 63(2-3), 282-289.

https://doi.org/10.7202/601423ar
Ce document est protégé par la loi sur le droit d'auteur. L'utilisation des services d'Érudit (y compris la reproduction) est assujettie à sa politique d'utilisation que vous pouvez consulter en ligne.

https://apropos.erudit.org/fr/usagers/politique-dutilisation/ 


\title{
Compte rendu de lecture
}

\author{
Georges DIONNE* \\ Université de Montréal
}

Essays On Economic Decisions Under Uncertainty, par JACQUES H. DRÈZE. - Cambridge University Press, 1987, 424 pages.

C'est avec beaucoup de joie que j'ai trouvé dans ma boîte aux lettres le dernier livre de Jacques Drèze. J'attendais ce livre depuis mon retour du CORE, en 1984. À cette époque, J. Drèze récrivait son essai sur les fondements du modèle d'espérance d'utilité (avec risque moral et préférences dépendantes des états de la nature) et envisageait de le diffuser dans un recueil avec d'autres articles déjà publiés sur l'incertain.

Cet essai est maintenant le chapitre 2 du livre que je vous présente. Le livre contient vingt textes sur l'économie de l'incertain écrits au cours des quelques vingt-cinq premières années de carrière de l'auteur. Après avoir lu certaines sections, j'ai décidé de parcourir tout le livre même si plusieurs articles m'étaient familiers. Je vous donne ici mes premières impressions; mon objectif est de faire ressortir les principales contributions à la littérature qui y sont publiées. Celles-ci peuvent être regroupées autour de cinq sujets : 1) les fondements de l'espérance d'utilité, 2) l'évaluation de la vie humaine, 3) les décisions de consommation, de portefeuille, d'offre de travail et d'assurance, 4) le comportement de la firme et 5) la caractérisation des propriétés de l'équilibre d'une industrie. Le livre contient sept sections qui ne correspondent pas aux cinq sujets de contribution mentionnés. Pour fin de présentation, il est plus utile de suivre les divisions de l'auteur.

\section{Prise de décision en incertitude : théorie générale}

Dans la première partie, Drèze présente cinq articles reliés à la théorie générale des choix en incertitude. Comme mentionné plus haut, un des essais est publié pour la première fois (chapitre 2). Il sera donc analysé plus en profondeur. Son contenu est intimement lié à ceux des chapitres 1,3 et 4 . Le premier chapitre

* Je remercie Danielle Blanchard et Camille Bronsard pour leurs commentaires sur une première version de ce texte. 
propose une revue, maintenant classique ${ }^{1}$, des fondements axiomatiques du théorème d'espérance d'utilité alors que le second est une traduction intégrale d'un article publié en 1961 dans La Décision. Celui-ci est, en quelque sorte, l'ancêtre du chapitre 2. Mais la traduction des « Fondements logiques de l'utilité cardinale et de la probabilité - subjective » n'est pas du tout superflue. Elle donne accès (auprès d'un public qui ne la connaissait pas) à une pièce maîtresse dans l'évolution historique de la réflexion sur les fondements de la prise de décision en incertitude. L'auteur analyse les effets de l'introduction simultanée du risque moral et des préférences dépendantes des états de la nature sur le modèle d'espérance d'utilité. Il est important de souligner que, dans son article de 1961, Drèze n'étudie pas de façon séparée les fondements du modèle d'espérance d'utilité en présence de risque moral et lorsque les fonctions d'utilité sont indépendantes des états de la nature. Il est principalement intéressé aux utilités dépendantes des états de la nature. En fait, il a besoin d'introduire des stratégies qui affectent les probabilités subjectives pour construire un indice d'espérance d'utilité lorsque les fonctions d'utilité sont dépendantes des états de la nature. Nous reviendrons sur cet aspect de sa contribution lorsque nous aborderons le chapitre 2.

Dès 1961, pour Jacques Drèze, introduire des stratégies qui affectent les probabilités des événements implique du risque moral car celles-ci ne sont pas observables. Même s'il est maintenant reconnu que c'est K. Arrow (1963) qui a été le premier à intégrer le risque moral dans la littérature économique, il est intéressant de souligner que, dans son article de 1961, Drèze avait bien identifié le problème d'information sans le définir explicitement. Des extraits de l'article original suffisent pour étayer le point :

«La difficulté que nous éprouvons à définir opérationnellement une relation de préférence entre les conséquences ... tient au fait, déjà signalé plus haut, que les états ne sont pas choisis par une personne au même titre que les biens. Il suffirait en effet que les états soient eux-mêmes des objets de choix pour que les conséquences le deviennent également; l'on pourrait alors postuler que les préférences entre les jeux induisent un ordre complet, et non seulement partiel, sur l'ensemble des conséquences.

Dans le modèle traditionnel, cette voie est fermée parce que les états et les événements sont défénis de manière à échapper complètement à l'influence de la personne... »

« Supposons que je m'intéresse à évaluer la probabilité subjective que Monsieur $X$ attache au fait que sa voiture soit volée dans les douze prochains mois. Qu'arrivera-til si j'offre à Monsieur $X$ un lot de Frs. 1,000,000, par exemple, en lui laissant le choix de faire dépendre ce lot, soit du vol de sa voiture dans les douze prochains mois, soit de l'événement complémentaire ? Évidemment, l'option choisi par Monsieur $X$ influencera nettement les précautions usuellles dont il entoure l'usage de son véhicule »...(Drèze, 1961, pp. 77-78).

1. Il est reproduit dans le recueil d'essais de Diamond et Rothschild (1978) (voir la bibliographie de l'introduction de ce recueil pour les principales références bibliographiques). 
Le chapitre 2 présente une formalisation détaillée et une extension de la contribution de 1961. Pour bien nous rendre compte de la difficulté de proposer des fondements théoriques au modèle d'espérance d'utilité lorsque les fonctions d'utilité sont dépendantes des états de la nature, abordons, pendant quelques instants, le modèle von Neuman-Morgenstern. Ce modèle est caractérisé par des probabilités objectives et des fonctions d'utilité indépendantes des états de la nature. De plus, il exclut les stratégies qui affectent les probabilités et, par conséquent, le risque moral.

Trois axiomes sont nécessaires à la construction du modèle d'espérance d'utilité : 1) ordre complet, 2) continuité et 3) indépendance. Le théorème de von Neuman-Morgenstern résulte de ces axiomes : la loterie $A$ sera préférée à la loterie B si et seulement si $E_{A} U>E_{B} U$.

La principale difficulté associée à l'introduction de probabilités subjectives réside dans l'identification de celles-ci et, par conséquent, dans la séparation de la fonction d'évaluation des loteries en deux composantes soit un indice d'utilité et une distribution de probabilités subjectives ${ }^{2}$. Afin d'obtenir une mesure de probabilité subjective unique et un indice d'utilité unique à une transformation linéaire positive près, des axiomes supplémentaires doivent être introduits. Parmi ceux-ci deux sont particulièrement importants pour notre discussion : le premier implique que les préférences sont indépendantes des états de la nature et le second, appelé « inversion d'ordre », élimine toute possibilité de risque moral.

Dans l'essai publié dans les premières pages de ce recueil, Drèze (1987) a présenté une extension au modèle avec probabilités subjectives afin d'introduire le risque moral. Sa méthodologie a consisté à proposer une version modifiée ou affaiblie de l'axiome « d'inversion d'ordre » en posant, comme hypothèse, que l'information a toujours une valeur non négative. Il montre qu'il est possible d'étendre le principe d'espérance d'utilité avec probabilités subjectives à des situations avec risque moral, c'est-à-dire pour lesquelles l'agent peut affecter les probabilités des événements par des stratrégies non observables. Il prouve qu'il existe un ensemble unique et convexe de probabilités associées à des stratégies non observables et un indice d'utilité unique à une transformation linéaire positive près. Cette démonstration est reprise dans la section 6 du chapitre 2 .

Il reste maintenant à introduire des utilités dépendantes des états de la nature. Drèze propose une extension du modèle de 1961 qui suggérait d'introduire des stratégies non observables afin que l'agent se « commette » sur des actes ce qui permet de réduire le problème d'identification simultanée des probabilités subjectives et des utilités associées aux états ${ }^{3}$.

2. Cette séparation est importante pour plusieurs applications économiques : modèles bayésiens, asymétrie d'information (sélection adverse et risque moral)...

3. Il est à noter qu'introduire des fonctions d'utilité dépendantes des états de la nature ne pose pas de problème particulier lorsque les probabilités des événements sont objectives et acceptées par tous les agents. 
Afin de bien visualiser la démarche de l'auteur, reprenons l'exemple qu'il nous propose. Un archer a pris la décision de participer à un concours olympique dans lequel ses chances de gagner sont bonnes. Deux événements sont possibles : gagner ou perdre le concours. Les indices d'utilité qui y sont associés diffèrent. Par exemple, gagner le concours fera de lui un archer célèbre. On lui demande d'indiquer ses préférences entre parier $\$ 100000$ sur gagner le concours (acte 1) ou parier $\$ 100000$ sur perdre le concours (acte 2). On lui indique également que c'est un jeu de hazard (pile ou face) qui déterminera si le pari sera sur gagner ou sur perdre et que cette détermination peut avoir lieu avant ou après le concours. Puisque nous sommes en situation de risque moral (c'est-à-dire que l'archer peut facilement perdre le concours si le pari, déterminé avant le concours, est sur perdre), celui-ci insiste pour que le jeu de pile ou face ait lieu avant le concours (sous l'hypothèse que la valeur de l'information est non négative). Supposons maintenant qu'il existe un troisième acte possible, soit parier $\$ 50000$ (acte 3) sur perdre, qui a les caractéristiques suivantes. Si un jeu de pile ou face doit déterminer si le pari est sur l'acte 1 ou l'acte 3, l'archer est indifférent sur le fait que la pièce de monnaie soit lancée avant ou après le concours; la même indifférence est exprimée si le jeu doit déterminer si le pari est sur l'acte 2 ou sur l'acte 3 .

Plusieurs conclusions peuvent être tirées de ces révélations de préférence. Elles permettent, entre autre, de déterminer les niveaux d'utilité conditionnels (aux états) en présence de risque moral. Par exemple, il est possible de calculer les écarts d'utilité entre gagner ou perdre pour tout niveau de richesse si plusieurs actes possibles sont offerts à l'agent décideur. Les préférences sur les actes révèlent celles sur les paires «prix-états ».

Dans la section 8 de son essai, Drèze montre formellement comment le risque moral permet de solutionner le double problème d'identification dans des situations caractérisées par des probabilités subjectives et des utilités dépendantes des états de la nature. Il montre que le problème est complètement résolu (l'ensemble convexe des probabilités est unique et les utilités sont uniques à une transformation linéaire positive près dans les états non nuls) si tous les états peuvent être influencés par les actions du décideur. Autrement, l'identification est partielle.

Le reste de l'essai est utilisé pour qualifier les résultats obtenus. Entre autre, l'auteur discute des problèmes d'idenfication dans les jeux contre la nature c'est-à-dire sans risque moral. Le récent livre de Karni (1985) résume bien cette approche. Elle consiste à définir des loteries auxiliaires avec probabilités objectives et à demander au décideur d'exprimer ses préférences sur des situations hypothétiques avec probabilités objectives. Ces révélations permettent d'identifier les goûts sur les paires prix-états et peuvent être utilisées, sous certains axiomes dont celui de «consistance forte», afin d'identifier les probabilités subjectives. De l'avis de J. Drèze la révélation des préférences doit être faite par le biais de choix entre des actes et non par des réponses verbales à des questions concernant des situations hypothétiques avec probabilités objectives. 
Il est difficile pour le lecteur de se faire une opinion rapide et de prendre partie. D'ailleurs, Drèze n'insiste pas. Il présente son approche comme une alternative qu'il qualifie:

" as a systematic exploration of the exact limitations of a fully behavioural theory of decision under moral hazard and state-dependent preferences » (p 69)

Le chapitre 4 est très important pour comprendre la portée de l'hypothèse que l'information a toujours une valeur non négative. En effet, après avoir présenté les grandes lignes de la mesure d'information proposée par Marschak, Drèze donne des situations ou des cas pour lesquels cette valeur peut être négative, d'où le terme « paradoxe » dans le titre. Ajoutons que le texte du chapitre quatre est aussi une traduction d'un texte publié en français en 1960.

Le contenu du chapitre 5 est plus récent (1981). L'auteur montre comment des choix d'assurance permettent de calculer des mesures implicites d'aversion au risque. Drèze obtient des mesures d'aversion au risque très élevées, ce qui est quelque peu surprenant.

\section{Marchés et prix}

La deuxième section du livre contient deux articles publiés respectivement en 1971 et 1979 . Le premier discute en détail des interprétations et implications du modèle d'Arrow (1953) sur l'allocation des ressources en incertitude. L'auteur montre, entre autre, que les prix des biens contingents ont les propriétés des probabilités des états de la nature tout en réflétant les raretés relatives des biens entre les états de la nature et les probabilités de réalisation. Cette investigation détaillée de la contribution d'Arrow (1953) n'est pas étrangère à l'importance qu'accorde Drèze aux modèles avec marchés contingents. Plusieurs articles subséquents à celui de 1971, de même que la préface du livre qui nous concerne, font largement référence à cette approche attribuée à Savage (1953), Arrow (1953) et Debreu $(1953,1959)$ :

"The common thread linking the essays in the present book is that the theory of individual decision, as developed for the "state " model by Savage, provides a starting point for research on the economics of uncertainty that is both natural and satisfactory. In particular it fits naturally into the general equilibrium model of allocation under uncertainty introduced by Arrow and developed by Debreu (1953, 1959) » (p. XVIII).

L'influence des travaux de ces trois auteurs est mise en valeur tout au long du livre.

Le second essai de la section II est une note technique qui montre que l'incertitude sur l'élasticité prix de la demande a un effet comparable à un coude dans la demande pour une entreprise riscophobe. 


\section{Décisions des consommateurs}

Les trois articles de cette section sont reliés à deux contributions importantes de l'auteur à l'économie de l'incertain: l'évaluation de la vie humaine en incertitude et la séparation des décisions de consommation, de choix de portefeuille, d'assurance et d'offre de travail en incertitude.

L'essai du chapitre 8 présente une analyse de la demande d'assurance et de la valeur de la sécurité lorsque les fonctions d'utilité sont dépendantes des états de la nature. Mentionnons immédiatement que, par hypothèse, les probabilités des événements sont connues objectivement ce qui évite plusieurs complications déjà mentionnées. L'intérêt des auteurs (Dehez et Drèze) est de tenir compte explicitement des désutilités autres que monétaires associées aux accidents dans une formule d'évaluation de projet public de sécurité. Ils obtiennent qu'une allocation efficace de sécurité publique est définie lorsque le coût marginal de sauver une vie est égal à la moyenne de ce que les membres de la société sont prêts à payer pour la sécurité (willingness-to-pay). Il s'agit d'une extension d'un premier article sur le sujet par Drèze (1962).

L'approche qui consiste à utiliser les dispositions à payer est maintenant bien acceptée dans la littérature. Jones-Lee qui a beaucoup contribué à l'acceptation de cette approche, est très explicite sur son origine. Nous reproduisons ici deux commentaires au sujet de l'article de Drèze (1962) :

"The paper by Drèze is without doubt one of the most important contributions to the literature on the value of safety » (p. 48) ... " this paper provides a foundation upon which an analysis can be built and chapter 5 of this book owes much to Drèze's ideas » (p. 49, Jones-Lee, 1976).

Il est difficile d'être plus explicite.

La question de la séparation des décisions des agents économiques en incertitude est encore d'actualité (voir l'introduction de ce recueil d'articles). Drèze et Modigliani y ont contributé dans deux articles publiés en 1966 et 1972; celui de 1966 incorpore les choix de temps de travail alors que celui de 1972 est limité aux choix de consommation, d'assurance et de portefeuille. L'article de 1972 contient plusieurs contributions. Trois d'entre elles retiendront notre attention. Drèze et Modigliani ont été les premiers à proposer une condition suffisante afin de séparer la décision de consommation présente des autres décisions. Dans un deuxième temps, ils ont généralisé les résultats de Leland et Sandmo et ont présenté une condition nécessaire et suffisante pour expliquer une augmentation de l'épargne des agents riscophobes suite à une augmentation du risque du revenu futur. La troisième contribution concerne la méthodologie utilisée dans l'analyse des effets de changement de risque. Il est intéressant de noter qu'une des étapes dans la preuve du théorème 4.1 (pages 198-199 et appendice C) est équivalente à l'étalement à moyenne constante de l'utilité proposé par Diamond et Stiglitz (1974). ${ }^{4}$

4. Voir Briys, Dionne et Eeckhoudt (1986) pour plus de détails. 


\section{Décisions du producteur}

Les chapitres 11, 12 et 13 étudient l'équilibre d'une industrie avec demande aléatoire. Le premier texte écrit en 1967 (avec J.J. Gabszewicz) a été généralisé par Drèze et Sheshinski en 1976 et 1984. Les auteurs étudient la nature de l'équilibre (efficacité, caractérisques, nombre de firmes...), son unicité et sa stabilité. Leur analyse présuppose que les entreprises maximisent l'espérance mathématique des profits et ont des anticipations communes sur la variation des prix. Dans la préface du livre, Drèze mentionne qu'il est possible de réinterpréter les résultats sous d'autres hypothèses de comportement des firmes. Ce qui nous permet d'introduire la section $\mathrm{V}$ du livre consacrée au comportement des entreprises en incertitude.

\section{Théorie de la firme}

Trois articles composent cette section. Le premier, publié en 1974, propose une extension des modèles de comportement de la firme en incertitude proposés indépendamment par Diamond et Stiglitz. Le modèle présenté incorpore des actifs financiers et discute des limites de l'assurance pour couvrir certains risques associés aux opérations des entreprises. Les deux autres contributions, publiées plus récemment $(1982,1985)$, discutent des différentes alternatives de comportement des entreprises mais, plus important encore, remettent en cause les modèles traditionnels et proposent une nouvelle approche générale de comportement des entreprises en incertitude.

Une première hypothèse de comportement est la maximisation de l'espérance mathématique de l'utilité des actionnaires. Si les marchés des capitaux sont parfaits et sans coûts de transaction, il est possible de montrer, qu'à l'équilibre, les préférences des actionnaires sont reflétées directement dans le prix des actions. Malheureusement, il est plutôt difficile de trouver des situations pour lesquelles ces conditions sont remplies. Certains auteurs ont proposé d'utiliser directement les préférences des actionnaires mais d'autres ont indiqué que cette approche impliquait des coûts de transaction importants et qu'il était préférable d'utiliser une fonction d'utilité de la firme. Drèze (1982) met en relief la nécessité de généraliser les théories existantes et suggère un modèle qui permet de tenir compte, à la fois, de plusieurs niveaux et de plusieurs critères de décision. A l'équilibre de ce modèle général, les décisions des entreprises devraient réfléter l'espérance mathématique de l'utilité des profits en terme d'une fonction d'utilité spécifique à la firme et qui peut dépendre des états de l'environnement, des préférences des actionnaires et des prix des actions dans les marchés financiers. Des étapes futures de recherche permettront d'évaluer l'applicabilité d'un modèle aussi général qui a l'originalité d'intégrer le comportement des entreprises en incertitude à celui des individus.

L'article de 1984 va encore beaucoup plus loin même s'il est plus près du fonctionnement observé des entreprises. Drèze propose un modèle qui tient compte des interactions entre les choix de portefeuille des consommateurs, les 
critères de décision des firmes et leurs formes de gestion. Il présente les entreprises comme des entités dirigées par un conseil de direction dont la composition reflète la propriété des actions et dont les décisions sont sujettes à des approbations par les actionnaires. Certaines décisions peuvent être déléguées à des administrateurs. Drèze étudie les conditions d'équilibre du modèle et montre qu'un équilibre existe sous une simple hypothèse de continuité.

L'auteur discute également des contrats de travail et de leur intégration dans le comportement général des entreprises; un sujet qu'il développe plus en détail dans un manuscrit contenant les leçons « Yrjö Jahnsson » qu'il a données en 1983. Mais la réflexion de l'auteur sur le fonctionnement du marché du travail en incertitude remonte à plus loin et elle a été l'objet de plusieurs séminaires.

\section{VI et VII. Capital humain, contrats de travail et décisions publiques}

Ces deux sections contiennent quatre articles. Trois d'entre eux reproduisent des interventions de l'auteur à titre de conférencier invité à différentes réunions scientifiques importantes soit la seconde conférence annuelle de l'Association de Genève (1979), la conférence Walras du congrès mondial de l'Association d'Économétrie (1975) et son discours présidentiel au congrès mondial de l'Association d'Économétrie (1972). Leur présentation ne contient pas d'analyse formelle et leur contenu résume la réflexion de l'auteur, accumulée au cours des années, sur des sujets aussi variés que le partage des risques et la demande de travail, les contrats implicites de travail, les compensations de chômage, les droits d'ancienneté, les entreprises autogérées, les choix de conditions de travail, la théorie de la décision et l'économétrie, etc. Finalement, le chapitre 19 (écrit avec $M$. Marchand) traite de la gestion des universités en incertitude.

\section{Conclusion}

Jacques Drèze a beaucoup apporté à l'analyse économique. Le livre que nous venons de présenter est limité à ses principales contributions à l'économie de l'incertain et de l'information. Il constitue une source très riche aux chercheurs intéressés à cette facette fondamentale de l'analyse économique. Il est à souhaiter que sa publication aidera à une plus grande diffusion et à une plus grande compréhension de l'oeuvre importante qu'il contient. 